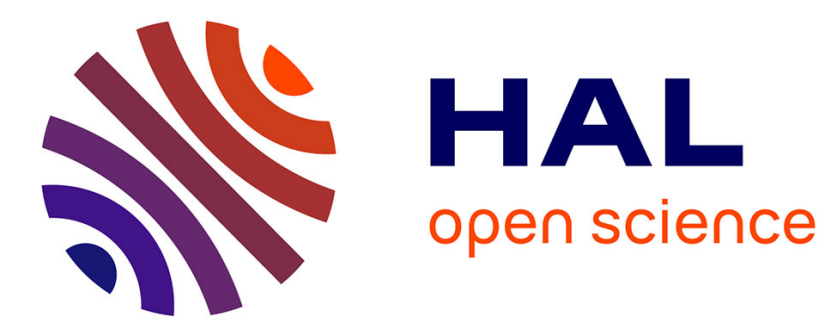

\title{
Une histoire de la méthode d'évaluation contingente
} Julien Milanesi

\section{To cite this version:}

Julien Milanesi. Une histoire de la méthode d'évaluation contingente. Genèses. Sciences sociales et histoire, 2011, 84 (3), 10.3917/gen.084.0006 . hal-01531153

\section{HAL Id: hal-01531153 \\ https://hal.science/hal-01531153}

Submitted on 12 Nov 2021

HAL is a multi-disciplinary open access archive for the deposit and dissemination of scientific research documents, whether they are published or not. The documents may come from teaching and research institutions in France or abroad, or from public or private research centers.
L'archive ouverte pluridisciplinaire HAL, est destinée au dépôt et à la diffusion de documents scientifiques de niveau recherche, publiés ou non, émanant des établissements d'enseignement et de recherche français ou étrangers, des laboratoires publics ou privés. 


\section{Une histoire de la méthode d'évaluation contingente}

Julien Milanesi, docteur en sciences économiques, Centre d'analyse théorique et traitement des données économiques (CATT), Université de Pau et des Pays de l'Adour.

Article à paraître dans la revue Genèses (revue AERES pour les domaines Sociologie-Démographie, Science Politique, Géographie-Aménagement-Urbanisme, Anthropologie-Ethnologie).

L'estimation du pretium doloris dans les tribunaux, la construction d'indicateurs de richesse, l'évaluation de la production domestique, la planification économique, la prise en compte de la dégradation des milieux ou de la santé des populations dans la décision publique sont autant de sujets qui peuvent poser la question de la mesure du prix de ce qui n'a pas de prix. Les économistes ont souvent pris cette interrogation comme une injonction à fournir des chiffres et donc à produire des outils d'évaluation. Ces outils font parfois leur chemin dans les cadres réglementaires jusqu'à revêtir tous les atours de la légitimité démocratique et scientifique, laissant ainsi croire que résoudre le questionnement initial n'était qu'une question de virtuosité technique. Derrière l'apparente naturalité du chiffre, se cache néanmoins la société en œuvre dans la genèse et la reconnaissance de ces méthodes et des chiffres qu'elles produisent. C'est ce que nous proposons d'illustrer ici à travers une histoire de la méthode d'évaluation contingente, un outil qui fut l'objet d'importantes controverses scientifiques mais dont l'usage s'est répandu dans la plupart des cadres réglementaires des pays industrialisés.

Initialement imaginée pour mesurer en terme monétaire la valeur de biens d'environnement (espèces protégées, écosystèmes, ...) ou de modifications de leur qualité (destruction, altération par pollution, ....), la MEC est également utilisée dans le domaine de la santé ou des risques technologiques - pour estimer par exemple la valeur de la vie humaine. Son principe est simple, il consiste à poser à un échantillon représentatif de la population une question de consentement à payer (ou à recevoir) pour accéder (ou renoncer) au bien à évaluer. La question est généralement posée en utilisant un support de paiement ad-hoc (taxe, cotisation à une association, etc), elle peut par exemple prendre cette forme: "Combien seriez-vous prêts à payer en impôts supplémentaires pour que la qualité de l'air s'améliore du niveau $x$ au niveau $y$ ?". Les réponses recueillies sont extrapolées au niveau de l'ensemble de la population concernée, ce qui permet d'obtenir le bénéfice total, mesuré en unités monétaires, de l'amélioration considérée (ici la qualité de l'air). Pour un bien naturel, comme une espèce protégée, les questions sont du même type et les résultats sont interprétés comme la valeur totale que les individus attribuent à ce bien.

Ce cadre d'interprétation des réponses des enquêtés est issu de l'Économie du bien-être et prend sa source dans l'utilitarisme Benthamien (Milanesi 2010). Bentham considérait à la fin du 
$18^{\text {ème }}$ siècle que les actions des individus ne peuvent être évaluées que relativement à l'utilité qu'elles leur apportent, qu'en fonction de leurs préférences individuelles. Les économistes néoclassiques, dans la continuité de cette pensée, établirent au $19^{\text {ème }}$ siècle que la valeur des biens ne prend sa source que dans l'utilité que ces biens apportent aux individus. Ils définirent ainsi l'hypothèse de la «valeur utilité» qui une fois étendue à la monnaie crée un système d'équivalence ou d'indifférence universel dans lequel tout peut théoriquement être évalué en terme monétaire (Hodgson, 1997). L'originalité de la méthode d'évaluation contingente est d'associer ce cadre théorique aux méthodes d'enquêtes par questionnaire en interprétant les réponses à des questions de consentement à payer (ou à recevoir) pour une variation de qualité (ou de quantité) de l'environnement comme la valeur économique qu'attribuent les enquêtés à ces variations. Le champ d'application de la méthode est ainsi théoriquement infini, elle peut potentiellement tout mesurer sous forme monétaire, ce qui constitue indéniablement une des clés de son succès.

Mais sa diffusion ne peut être comprise sans considérer la demande croissante émanant de la société d'intégration de l'environnement dans la décision économique publique et privée. Elle s'inscrit également dans un climat idéologique et politique de confiance envers les modes de régulation marchands, identifiant les problèmes d'environnement à des dysfonctionnements ou des incomplétudes du marché et où «il faut donner un prix à la nature pour la protéger »'. La MEC est ainsi un objet scientifique hybride (Latour 1997) dont le développement ne peut s'expliquer qu'en retraçant l'imbroglio administratif, scientifique, politique, social et idéologique que fut son histoire depuis son invention en 1952. C'est ce que nous ferons ici en distinguant trois périodes: sa genèse aux États-Unis entre les années 50 et 80, son arrivée à «maturité » dans les années 90 et son actualité.

\section{La genèse (1952-1989) - Une histoire américaine}

La genèse de la MEC s'étend sur un peu plus de 35 ans entre son «invention » par Ciriacy Wantrup en 1952 et sa «stabilisation» par Mitchell et Carson en 1989. Entre ces deux dates, les auteurs ayant travaillé à son développement lui donnèrent un cadre théorique et définirent la plupart des enjeux méthodologiques. La méthode connut dans le même temps sa première reconnaissance et légitimation publique. L'étude de ces premières années d'élaboration de l'évaluation contingente montre que le développement scientifique de la méthode et celui de son usage public sont intimement liés et s'inscrivent dans un territoire particulier: les États-Unis d'Amérique. 


\section{Prémisses et période exploratoire}

L'invention et le développement de la MEC sont très étroitement liés à la montée en puissance de l'analyse coût-bénéfice dans la décision publique (Ackerman 2004). L'objectif de l'ACB est de rationaliser et d'optimiser la décision en la fondant sur une évaluation qui se veut objective des coûts et des bénéfices. L'ACB pourrait être qualifiée de méthode d'optimisation par le marché, ou de rationalisation marchande, car elle associe la volonté de prendre les meilleures décisions possibles - sans gaspillage d'argent public - à l'idée que le modèle du marché est le plus efficace pour parvenir à cet objectif.

Alors qu'elle se limitait à une simple analyse comptable dans les années 50, l'ACB a peu à peu cherché à évaluer la satisfaction (ou l'insatisfaction) finale que tirerait un groupe d'individus d'un projet ou d'une politique publique. Le problème essentiel d'une ACB ainsi définie est de mesurer les bénéfices ou les coûts induits situés hors de la sphère marchande. En matière d'environnement, ces gains ou pertes furent initialement mesurés à l'aide des méthodes dites des «coûts de transport » puis des «prix hédoniques » (Randall 1998) qui sont basées sur les «préférences révélées » par les individus, c'est à dire sur des choix déjà effectués sur des marchés réels (transport ou immobilier). Ces méthodes ont néanmoins comme défaut de ne pas pouvoir s'appliquer à tous les biens et surtout, de ne pas mesurer ce que les théoriciens néoclassiques de l'environnement qualifient de «valeur de non-usage » («non use value »). ${ }^{\text {ii }}$

C'est dans ce contexte que Ciriracy-Wantrup, un économiste de l'université de Berkeley, inventa en 1952 la MEC qui est fondée sur les «préférences déclarées » par les individus sur des marchés virtuels. Sans développer lui-même cette voie, il ouvrait un nouveau champ de recherche dont l'originalité résidait dans la rencontre entre théorie de l'économie du bien-être et apports des méthodes d'enquête par entretien ou questionnaire et qui permet de proposer une mesure de la valeur d'existence de biens non marchands. C'est cette caractéristique qui fit, et qui fait encore, son succès par rapport à d'autres méthodes.

La littérature retient généralement l'étude de Robert Davis sur les forêts du Maine, publiée dans son doctorat en 1963 comme la première application de la méthode. Les premières critiques vinrent en 1965 par Anthony Scott qui, en attaquant l'évaluation contingente sur son caractère hypothétique, inaugurait une longue série de remises en cause: «Posez une question hypothétique et vous obtiendrez une réponse hypothétique (...) les résultats de cette procédure [d'évaluation contingente] ont conduit à des évaluations extrêmement fantaisistes » (Mitchell et Carson 1989: 172). Le nombre de ces évaluations se multiplia néanmoins dans les années suivantes aux ÉtatsUnis à des fins exploratoires et méthodologiques et sur une grande variété de sujets: pollution de 
l'air, risques médicaux, droits de chasse, congestion des chemins pour promeneurs, aménités de parcs en zone urbaine, programmes réduisant le risque de mort par attaque cardiaque, amélioration de la qualité de l'eau des plages, diminution du risque de mortalité en cas d'accident nucléaire, etc. (ibid.: 10-13).

\section{Consolidation théorique et premières consécrations juridiques}

Les années 80 furent celles de la consécration juridique et scientifique de l'évaluation contingente. Dans cette période de poussée des idées conservatrices et de foi dans la régulation marchande, l'évaluation contingente était un outil qui, par sa faculté à créer des marchés là où ils n'existaient pas, ouvrait d'immenses possibilités. L'administration étasunienne du président Reagan eut ainsi une influence décisive sur le développement scientifique et juridique de la méthode.

Appuyée notamment par Robert Davis, entre temps recruté au Department of Interior (Loomis, 1999: 613), la MEC fut intégrée à la fin des années 70 aux méthodes recommandées pour les évaluations de projets de l'administration étasunienne. Son usage fut ensuite promu en 1981 par une ordonnance exigeant l'usage d'ACB dans l'évaluation des principales nouvelles réglementations fédérales. Du fait de cette nouvelle loi, l'Environmental Protection Agency commanda de nombreuses enquêtes d'évaluations contingentes (ibid. : 616-617).

Suite à l'adoption du Comprehensive, Environmental Response, Compensation and Liability Act (CERCLA) la MEC fut également intégrée aux méthodes d'évaluation des dommages définies par le Department of Interior en 1986, mais dans un rôle secondaire qui fut contesté devant les tribunaux par l'Etat du Ohio (Thompson: 1992). La cour d'appel du district de Columbia donna raison à ce dernier en 1989, en indiquant que la MEC était la «meilleure procédure disponible» pour évaluer les dommages devant les tribunaux (Navrud et Pruckner 1997: 11).

Un dernier champ d'intervention de la MEC dans le cadre réglementaire étasunien est celui de l'évaluation des externalités ${ }^{\mathrm{iii}}$ produites par les centrales électriques, qui est exigé dans l' Electric Consumer Act adopté en 1986 (Loomis 1999: 617-618).

Ce nouvel environnement législatif suscita un besoin d'enquêtes de terrains et de consolidation théorique de la méthode. L'administration étasunienne, surtout par l'intermédiaire de l'Environmental Protection Agency (EPA), joua également un rôle important dans ces développements scientifiques qui virent émerger au cours des années 80 un corpus méthodologique et théorique synthétisé dans les deux premiers ouvrages sur la MEC, parus en 1986 et 1989.

Le premier fut celui dirigé par Cummings, Brookshire et Schulze et publié en 1986, il faisait suite à une conférence organisée en 1984 par l'EPA. Le second, de Robert Mitchell et Richard Carson, est l'ouvrage de référence sur la MEC. Édité en 1989 ce livre s'est notamment nourri de 
leurs travaux sur l'eau également financés, dès 1981, par l'EPA. Après environ 30 années de recherche, cette publication marquait une étape importante dans le développement de l'évaluation contingente. Ses fondements théoriques y étaient stabilisés, plus de 100 enquêtes y étaient recensées et les auteurs y donnaient des conseils de méthode pour conduire de bonnes évaluations contingentes. Des questions subsistaient mais la légitimité scientifique de la méthode, renforcée par la caution du cadre réglementaire étasunien, n'était plus questionnée par les spécialistes de l'exercice.

\section{Les travaux pionniers de la banque mondiale dans les PED}

Alors que les enquêtes d'évaluation contingente se multipliaient au États-Unis, les années 80 furent celles du début de la diffusion de la méthode sur les autres continents. Stale Navrud recensait en 1992 environ deux cents enquêtes conduites en Europe (Navrud et Vagnes 2000). De premières enquêtes ont également été menées dans des pays en développement, essentiellement par des chercheurs étasuniens.

La première d'entre elles a été réalisée en Thaïlande (Grandstaff et Dixon 1980), mais la littérature a surtout retenu les travaux de Dale Whittington qui, après une tentative infructueuse de la Banque mondiale dans les années 70 (Briscoe et al. 1990: 119), fut le premier, à Haïti en 1986, à conduire une enquête sur la demande en eau en milieu rural (Whittington et al. 1990). Cet auteur, par sa production abondante, ses collaborations institutionnelles et la dynamique qu'il initia au sein de son université de Chapell Hill (Caroline du Nord) eut une influence fondamentale sur la diffusion des enquêtes de consentement à payer (CAP) dans les pays en développement (Milanesi 2007: 38, 44 et 98). Il dirigeait lors de l'étude réalisée à Haïti un projet « Eau et assainissement pour la santé $»^{\text {iv }}$ financé par USAID et rédigea alors pour l'organisme de coopération étasunien le premier guide de mise en œuvre d'une évaluation contingente dans les PED (WASH 1988). Il devint par la suite le co-directeur de l'équipe de recherche de la Banque mondiale sur la demande en eau (World Bank Water Demand Research Team 1993).

Marquée par la même confiance dans les modes de régulation marchands que l'administration américaine, la Banque mondiale a développé l'usage de la MEC afin de rapprocher les projets d'adduction d'eau d'une certaine "vérité des prix", gage de meilleure efficacité. L'objectif était alors de mesurer la demande des ménages pour de nouveaux services à l'aide d'enquêtes de CAP, et d'apporter ainsi des informations sur la faisabilité et la pérennité financière des projets.

Ce fut ainsi une nouvelle page de l'histoire de la MEC qui s'ouvrit avec ces travaux et qui fut essentiellement empruntée dans les pays du Sud. Cet objectif opérationnel de faisabilité des projets est en effet de tout autre nature que le critère de légitimité de politiques publiques que cherche à 
apporter l'analyse coût-bénéfice (Milanesi 2007: 100).

\section{Le temps des débats (les années 90)}

A la fin des années 80, la MEC semblait avoir gagné sa légitimité publique et scientifique. Cette réussite masquait néanmoins un confinement des débats dans des cercles d'économistes initiés et l'absence d'un véritable "baptême du feu" où elle serait utilisée pour évaluer des bénéfices ou des dommages importants. Celui-ci advint avec la marée noire causée par le naufrage de l'Exxon Valdez qui plaça l'évaluation contingente au centre d'enjeux économiques gigantesques. Sa fiabilité fut très fortement mise en question dans des débats largement diffusés et impliquant plusieurs lauréats (et futurs lauréats) du prix de la Banque de Suède en mémoire d'Alfred Nobel.

Le début des années 90 fut donc, aux États-Unis, la période de popularisation de la MEC dans les milieux académiques. Ce fut aussi celle de sa consolidation et banalisation juridique.

\section{Le naufrage de l'Exxon-Valdez}

La MEC était entrée une première fois dans le débat public lors de la contestation des règles de mesures adoptées par le Department of Interior (DoI) pour le CERCLA. Ce débat rebondit en mars 1989 avec le naufrage du pétrolier "Exxon Valdez", du groupe Exxon, dans le détroit du Prince William en Alaska. Ce qui avait pu apparaître jusque-là comme un simple enjeu scientifique devenait, du fait de l'ampleur de la catastrophe, un enjeu économique gigantesque. L'État d'Alaska et le gouvernement fédéral des États-Unis déposèrent en effet une plainte et, afin de chiffrer les dommages, l'État d'Alaska commanda une étude utilisant une enquête d'évaluation contingente qui chiffra à 3 milliards de dollars les pertes de valeurs de non-usage (Carson et al. 1992) ${ }^{\mathrm{v}}$. Exxon finança une contre expertise qui mesura les pertes d'usages en utilisant la méthode des coûts de transport et qui conclut à une perte de 5 millions de dollars (Hausman et al. 1995), soit un montant 600 fois inférieur à celui de l'enquête par évaluation contingente. Exxon fut finalement condamné au pénal et au civil et eut à payer, à travers divers arrangements passés en 1991 avec le gouvernement fédéral et l'Etat d'Alaska, un montant total de 2,1 milliards de dollars.

Le naufrage de l'Exxonon Valdez eut également une conséquence juridique de plus longue portée.e. Suite à ce désastre le Congrès des États-Unis décida de légiférer sur les marées noires. Il adopta en 1990 le Oil Pollution Act qui, à côté de mesures destinées à réduire la probabilité de futures catastrophes, devait clarifier les procédures d'évaluation et de compensation des dommages. L'élaboration de ces règles fut confiée au National Oceanic and Atmospheric Administration $(N O A A)$ qui constitua un panel d'experts chargé de répondre à la question suivante: la méthode 
d'évaluation contingente peut-elle fournir une estimation de la valeur d'existence ou de non-usage suffisamment fiable pour être utilisée dans l'évaluation des dommages environnementaux? Il est intéressant de noter avec Paul Portney (1994), membre du Panel, qu'il n'était pas demandé à ces experts de donner leur avis sur la validité du concept de valeur d'existence ou de non-usage.

La légitimité de ce panel était fondée par la direction qu'en assuraient conjointement Kenneth Arrow et Robert Solow, tous deux titulaires du «prix Nobel » d'économie. L'évaluation contingente, jusque-là objet de recherche de quelques économistes de l'environnement, fut ainsi placée pendant quelques temps au centre de débats académiques impliquant des économistes parmi les plus reconnus aux États-Unis (Portney 1994). L'administration américaine était engagée dans ce débat à travers le NOAA, le milieux des affaires également, surtout à travers Exxon qui finança un nombre important de travaux.

Le premier de ces travaux, qui fait depuis référence dans la critique de la MEC, est l'ouvrage édité sous la direction de Jerry Hausman, suite à un séminaire organisé à Washington DC les 2 et 3 avril 1992. La très grande majorité des contributions qui y furent présentées étaient "à charge" (et issues de travaux financés par Exxon), mais les débats impliquèrent également de nombreux praticiens et défenseurs de l'évaluation contingente.

\section{La crédibilité de la MEC remise en question}

Les contributeurs au séminaire apportèrent plusieurs niveaux de critiques traduisant majoritairement une méfiance envers les données récoltées dans un contexte hypothétique. Dans la lignée de Scott en 1965, de nombreux économistes pensent en effet qu'une question hypothétique ne peut apporter que des résultats hypothétiques, les seules données fiables, crédibles, sont celles récoltées à partir de transactions ayant déjà eu lieu.

Diamond et Hausman (1993, 1994) développèrent cette critique, et définirent trois types de tests "internes" à la méthode. En l'absence de référence dans le réel pour faire des comparaisons (il n'y a pas de marché réel, par définition, pour les biens non marchands) les réponses récoltées devraient d'après eux être évaluées en fonction de leur précision, de leur crédibilité et de leur fiabilité. La précision fait référence à la variabilité des réponses, qui peut être améliorée en augmentant la taille de l'échantillon d'enquête, ils évacuèrent donc ce point pour se concentrer sur les deux autres.

Le test de crédibilité doit permettre de répondre à cette question: "les individus répondent-ils à la question qui leur est posée?". Diamond et Hausman affirmèrent que non en identifiant, à l'aide de travaux expérimentaux d'économie comportementale, cinq hypothèses alternatives de réponse à une question de CAP (Diamond et Hausman 1994: 47). La première hypothèse, la seule « valable », 
c'est à dire en conformité avec la théorie sous-jacente à la MEC, est que les individus font un arbitrage (un exercice de substitution) entre le bien considéré et leur revenu. Selon les quatre autres hypothèses, les réponses récoltées dans le cadre d'enquêtes de CAP traduisent (1) une attitude relative à un bien public et non au bien en question (Kahneman et Ritov 1994), (2) un sentiment de satisfaction morale (Kahneman et Knetsch 1992), (3) un calcul coût-bénéfice personnel concernant le projet ou la réglementation en question (Schkade et Payne 1993) ou bien (4) une réaction cherchant par exemple à «punir » le pollueur. Si ces quatre dernières alternatives représentent une large majorité des réponses recueillies (comme dans l'étude de Schkade et Payne (1993)), les chiffres sont inutilisables pour la décision publique et cela remet fondamentalement en question l'utilité de la MEC.

Au delà de cette conclusion pratique, ces résultats posent plus globalement la question de la robustesse du cadre théorique issu de l'économie du bien être. Des échanges eurent lieu à ce sujet (Milanesi 2007 : 60-61), mais la défense de la MEC, comme il est courant dans les débats sur la méthode, s'exerça surtout sur un plan méthodologique (Hanemann, 1994). Ceci revient à interroger non plus la crédibilité mais la fiabilité de la méthode. Les possibilités de biais sont en effet nombreuses (Venkatachalam 2003) mais la littérature considère généralement que ceux-ci sont évitables, ou limités, si l'enquête est correctement conduite. Ce fut également la position du panel réuni par le NOAA qui confirma ainsi l'usage de l'évaluation contingente dans la juridiction étasunienne.

\section{Légitimation et banalisation de la méthode}

Le panel d'experts réuni par le NOAA rendit ses conclusions le 11 janvier 1993. Selon Paul Portney (1994: 8-10) celles-ci ne satisfirent personne. Les opposants à la méthode furent déçus par la conclusion selon laquelle «les enquêtes d'évaluation contingente peuvent produire des estimations suffisamment fiables pour servir de point de départ aux processus judiciaires d'évaluation des dommages, pertes de valeurs de non-usage y compris » (Arrow et al. 1993: 42), tandis que ses défenseurs, bien que satisfaits de cette reconnaissance, furent contrariés par les restrictions qu'imposait le Panel dans son usage. En effet, d'après Portney, cette conclusion fut atteinte avec une réserve unanime des membres qui étaient peu convaincus de la fiabilité des enquêtes menées jusqu'alors et qui reconnaissaient la pertinence de certaines objections (ibid. 1993: $6)$.

Le Panel définit ainsi un guide de méthode assez strict que devraient respecter les applications futures afin que les résultats soient jugés recevables devant les tribunaux (voir Encadré 1 ci-dessous), il établit également une série de tests de cohérence interne permettant de conclure sur 
la crédibilité des réponses apportées et, pour limiter les risques de surestimation des valeurs, conseilla de diviser par deux les montants obtenus (ibid. 1993). Toutes ces précautions devaient conduire à des évaluations contingentes "idéales" mais elles ont l'important défaut de rendre les enquêtes très compliquées et coûteuses.

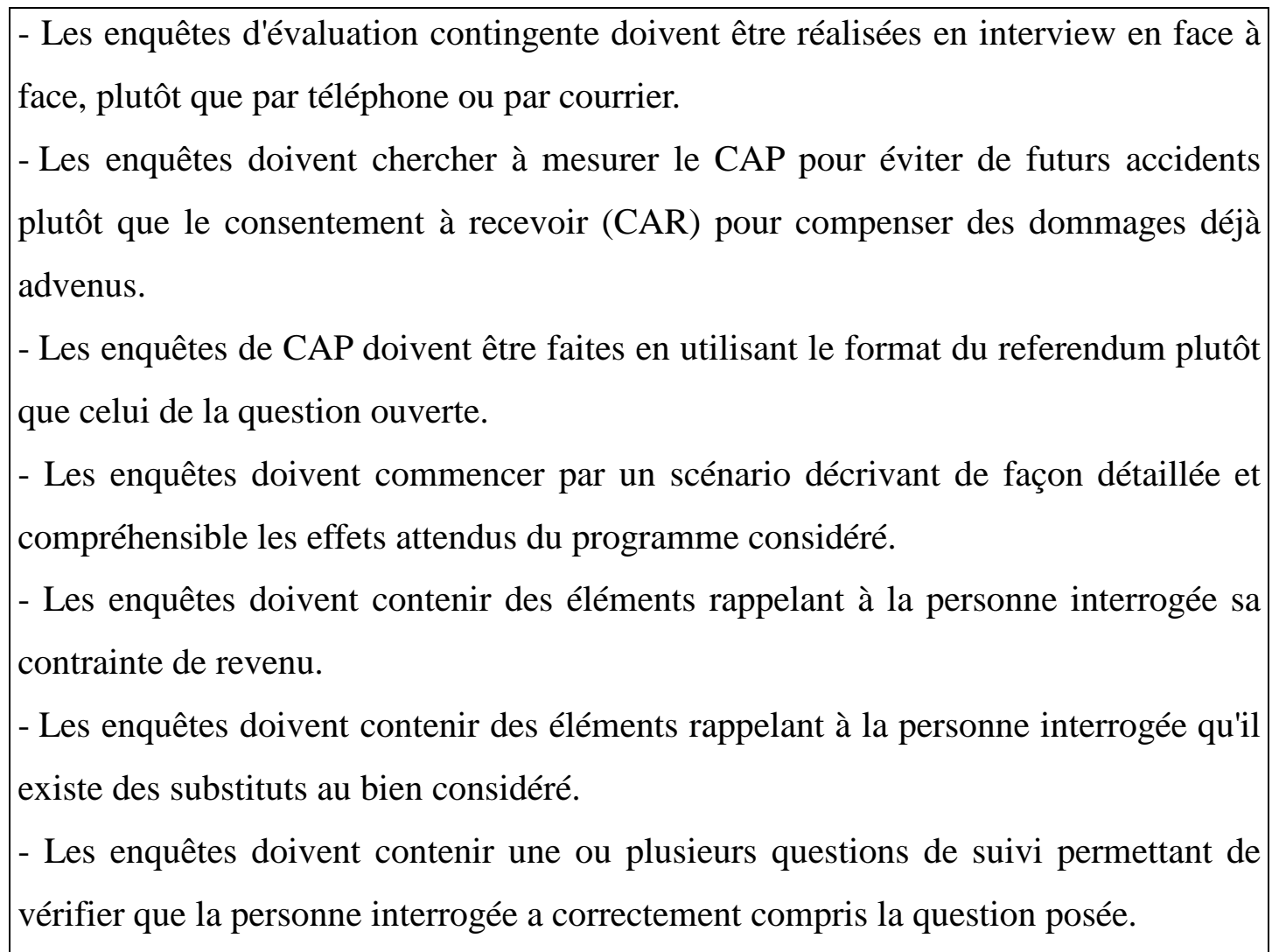

Encadré 1: Les sept recommandations les plus importantes du Panel constitué par le NOAA (Portney, 1994, 9).

Ces conclusions n'éteignirent pas la controverse, Diamond et Hausman (1994: 61-62) critiquèrent notamment la défense «méthodologique» de la MEC, en arguant que le Panel ne prouvait nulle part que le respect strict de leur guide de méthode conduisait à des résultats fiables. Ces objections furent plutôt vaines car, bien que les réserves soient nombreuses dans leur rapport, l'avis positif donné par le panel du NOAA apporta une nouvelle légitimité à la méthode.

Le NOAA suivit largement les recommandations du Panel dans l'établissement, en janvier 1996, de ses règles d'évaluation des dommages de ressources naturelles. Mais le rôle des méthodes d'évaluation y fut diminué au profit d'une logique de restauration des biens endommagés (Navrud et Pruckner 1997: 13), et la MEC, légitimée mais banalisée, fut rangée parmi d'autres méthodes d'évaluation (Thompson: 1992).

Cette banalisation ne saurait néanmoins diminuer l'importance du chemin parcouru : en quelques années, la MEC est passée du statut de méthode quasi inconnue et controversée, à celui d'objet de débat passionné, pour être finalement un outil commun de politique publique. D'aucuns 
pourront y voir la trajectoire rectiligne d'un objet scientifique qui, par la vertu du débat entre spécialistes, se perfectionne et atteint une légitimité suffisante pour être incorporé à la décision publique. Averti par Bruno Latour (1997), on peut plutôt y voir un intéressant spécimen d'hybride qui, à la rencontre des sphères scientifiques et politiques, fut légitimé par un étonnant aller-retour des formes de justification. Après s'être développée dans la sphère scientifique grâce à la légitimation et le support de la l'administration étasunienne, la MEC s'est en effet finalement imposée dans la sphère de la décision publique grâce à la légitimation scientifique d'économistes laissant de côté les objections théoriques de fond pour adopter une attitude pragmatique que Paul Portney (1994: 16) résume ainsi: «Que les économistes soient d'accord ou pas, il me semble inévitable que la méthode d'évaluation contingente jouera un rôle dans la formulation des politiques publiques. (...) Il est sûrement mieux pour les économistes qu'ils soient inclus à tous les niveaux de débats que de rester en marge et laisser d'autres décider de la manière dont sera utilisé cet outil. »

\section{Usages et diffusion institutionnelle récente}

Durant ces débats aux États-Unis la MEC s'est diffusée à travers le monde, dans les pays industrialisés (dont les pays européens) à travers l'extension de l'analyse économique de type coûtbénéfice dans la formulation des politiques publiques, et dans les pays du Sud en accompagnant le mouvement de privatisation de nombreux services. La prise de conscience des crises écologiques globales lui a également donné une nouvelle actualité.

\section{L'arrivée en Europe}

En Europe, bien que réelle au début des années 2000, la diffusion de l'évaluation contingente eut plus de mal à se faire qu'aux États-Unis (Bonnieux et Rainelli 1999: 593-594). Le conséquentialisme utilitariste qui sous-tend l'analyse coût-bénéfice et l'évaluation monétaire des biens environnementaux a eu du mal à s'imposer dans des pays aux traditions philosophiques plutôt déontologiques.

Le Royaume-Uni, terre de naissance de l'utilitarisme, fait néanmoins figure d'exception. Il fut le premier pays européen à utiliser l'évaluation contingente (en 1971), dans une analyse coûtbénéfice portant sur le troisième aéroport londonien (ibid.) et en 2000 Navrud et Mette recensaient 192 enquêtes d'évaluation monétaire de la nature conduites dans ce pays entre 1992 et 1999 sur un total de 450 sur toute l'Europe. Sur un plan réglementaire, ces méthodes ont fait leur entrée dans les politiques publiques anglaises en juillet 1989 avec Chris Patten, nouveau secrétaire d'Etat à l'environnement du troisième gouvernement Thatcher, et son conseiller David Pearce, économiste et 
spécialiste de l'analyse coût-bénéfice et des méthodes d'évaluation (Grove-White 1997).

La MEC est arrivée plus tardivement en France, la première étude a été conduite en 1990 sur des zones humides (Bonnieux et Rainelli 1999: 598). La production française d'évaluations contingentes n'a pas été exactement chiffrée mais Touaty et al. estimaient en 2004 à une centaine, toutes méthodes confondues, le nombre d'études de valorisations monétaires de biens environnementaux ou de santé.

Concernant la réglementation, «en 1996, le Conseil scientifique de l'Institut français de l'environnement (Ifen) avait conclu que le contexte français était globalement peu favorable à l'évaluation des dommages environnementaux et avait fait des recommandations pour améliorer cette situation» (Touaty et Gié 2004: 3). L'usage des méthodes d'évaluation monétaire de l'environnement, et donc de la MEC, s'est surtout développé depuis dans l'évaluation de projets, en matière d'infrastructures de transports (Ministère de l'équipement 2005, Baumstark 2004) ou dans le domaine de l'eau, dans le cadre de l'application de la directive cadre Européenne (2000/60/CE du 23 octobre 2000). Le ministère chargé de l'écologie développe et soutient ainsi depuis le début des années 2000 des travaux sur l'évaluation des dommages et bénéfices environnementaux dans ce domaine qui ont notamment abouti à la création d'un site internet (http://www.economie.eaufrance.fr/). Ce site contient une base de donnée d'études d'évaluation de dommages et de bénéfices (dont de nombreuses évaluations contingentes) qui avait été initiée en 2003 par des chercheurs de l'INRA (Amigues et alii 2003).

Le soutien du Ministère de l'écologie à l'évaluation monétaire de l'environnement s'est affirmé depuis le début des années 2000, il la considère à présent comme « une étape essentielle dans le choix de projets et la distribution des politiques publiques » (MEDD 2003). Cet engagement s'est concrétisé par la publication en 2005 de "guides de bonnes pratiques" sur les différentes méthodes, dont la MEC (Terra 2005), et par la réalisation de différentes études faisant appel à la MEC (ou à l'analyse conjointe ${ }^{\mathrm{vi}}$ ) s'inscrivant souvent dans des analyses coûts-bénéfices ${ }^{\mathrm{vii}}$. Un rapport a également été commandé en 2008 par le Premier Ministre au Centre d'Analyse Stratégique sur la valeur de la biodiversité et des services liés aux écosystèmes (Chevassus-au-Louis et al. 2009).

L'évaluation contingente, à l'image de l'ensemble des méthodes d'évaluation des bénéfices, a également mis du temps à s'imposer dans la législation européenne (Bonnieux et Rainelli 1999, Grasso Pareglio 2002). Quasi inexistantes jusqu'au début des années 90 ces méthodes apparurent comme un moyen de répondre à l'injonction du traité de Maastricht de spécifiquement considérer les coûts et bénéfices potentiels des politiques environnementales de l'UE, puis furent intégrées plus globalement dans les analyses d'impact des nouvelles réglementations (Milanesi 2007 : 77-81). 
Nous avons vu qu'elles tiennent également une place importante dans l'application de la directive cadre sur l'eau adoptée en 2000.

\section{Nouvelles routes}

Les travaux de la Banque Mondiale développés dans les années 80 dans les pays du Sud, consistant à utiliser la MEC pour mesurer la demande pour de nouveaux biens ou services, ouvrirent dans les deux décennies suivantes un champ d'étude et de recherche considérable, alimenté par la privatisation ou la recherche de l'équilibre financier des structures de gestion des services d'eau, d'assainissement ou de santé. Ce nouveau paradigme de mise en cuvre des projets de développement a généré entre 1989 et 2000 la production d'enquêtes d'évaluation contingente ou de guides d'application de la méthode par quasiment toutes les principales organisations internationales ou nationales d'aide et de développement (Milanesi 2007: 91-92). Cet effort a essentiellement consisté en un perfectionnement de l'outil d'enquête de CAP et de l'analyse statistique des résultats. Une littérature abondante s'est ainsi constituée, avec ses propres problématiques et questionnements méthodologiques, éloignant petit à petit ces mesures de demande de la méthode d'évaluation contingente. La plupart des auteurs n'allèrent néanmoins pas jusqu'à abandonner les fondements théoriques de la MEC issus de l'économie du bien-être, pourtant devenus inutiles. Ces enquêtes pourraient pourtant gagner en fiabilité par cet abandon et leur transformation en «mesure contingente de la demande » (ibid. : 268).

Les années 2000 furent par contre celles d'un nouvel élan, porté par la prise de conscience des crises écologiques planétaires, dans ce qui constitue l'usage originel de la MEC: l'évaluation monétaire des dommages ou bénéfices environnementaux non marchands. Associés à d'autres types de mesures ces travaux ont été largement mobilisés pour fournir des évaluations globales, se voulant exhaustives, de la valeur des écosystèmes (Évaluation des écosystèmes pour le millénaire initiée par l'ONU en 2001, initiative internationale sur «L'Économie des écosystèmes et de la biodiversité » (Sukhdev 2008)) ou des coûts du changement climatique (rapport de Nicolas Stern (2006)).

Ces nouveaux besoins ont créé une demande croissante en enquêtes d'évaluation contingente. Leur mise en œuvre dans les «règles de l'art» définies par le Panel du NOAA est cependant très coûteuse. Leur coût est généralement supérieur aux bénéfices qu'apporte l'information recueillie par l'enquête et donc, en toute rationalité, les institutions préfèrent à présent retenir les valeurs obtenues à l'aide d'enquêtes déjà réalisées dans des contextes différents (Amigues et alii 2003). Ces transferts de valeurs posent d'importants problèmes de fiabilité (Chevassus-auLouis et al. 2008: 189-192) mais leur usage va grandissant grâce au développement de bases de 
données d'enquêtes accessibles sur Internet. L'Environmental Valuation Reference Inventory (EVRI, www.evri.ca) créée à l'initiative du Canada et rejointe par la France, la Grande-Bretagne et les États-Unis rassemble par exemple en 2010 plus de 2000 enquêtes (sur l'air, l'eau, les animaux, les plantes, etc.) dont 1123 conduites avec des méthodes fondées sur les préférences déclarées. D'autres initiatives du même type et de moindre ampleur ont été développées à l'échelle nationale (comme sur l'eau en France) ou internationale (ibid. : 193).

\section{Le renouvellement critique}

La diffusion de la MEC dans les années 90 et 2000 a été accompagnée, particulièrement en Europe, d'un renouvellement de la critique. Plutôt que de s'attacher à démontrer le mauvais fonctionnement de la méthode, comme cela avait été fait durant la controverse ayant suivi le naufrage de l'Exxon Valdez, cette critique, que l'on peut qualifier d'externe, s'est attaquée à ses fondements philosophiques et théoriques. Elle est venue de philosophes, d'économistes institutionnalistes et du courant, récemment né, de l'Économie écologique.

Des philosophes, aux premiers rangs desquels on trouve Mark Sagoff (2004) et John O'Neill (1997), sont revenus sur les fondements utilitaristes de la méthode pour souligner, dans la tradition déontologique, que les individus n'agissent pas seulement pour satisfaire leurs préférences personnelles mais qu'ils se conforment également à des injonctions morales. O'Neill (1997: 77) souligne le pluralisme des valeurs existant sur les problèmes d'environnement et juge ces valeurs irréductibles les unes aux autres. Il introduit ainsi des situations d'incommensurabilité, où toute évaluation sous une unité de mesure commune est impossible.

Les économistes institutionnalistes ont repris cette critique philosophique en insistant sur l'importance des comportements moraux dans la décision mais ont également porté la critique sur le cadre théorique issu de l'Économie du bien-être en insistant sur le fait que les préférences des individus sont fortement influencées par le contexte de la décision et ne peuvent donc être considérées comme stables (Hodgson 1997). Cette critique trouve des échos dans les travaux expérimentaux d'économie comportementale présentés par Diamond et Hausman (1993) lors du débat aux États-Unis. Un des résultats de ce courant de recherche récent est la mise en évidence du caractère procédural et contextualisé de la décision des individus (Tversky et Thaler 1990). Si ces conclusions sont robustes on comprend bien la difficulté d'utiliser pour des décisions publiques des mesures qui n'auraient pas un caractère invariable. Les travaux d'économistes institutionnalistes sur la monnaie (Aglietta et Orléan 2002 ) offrent également une ressource critique importante en définissant l'espace marchand comme un espace limité, circonscrit (Milanesi 2007: 185).

Ces différents arguments ont été abondamment repris par des auteurs appartenant au courant 
de l'Économie Écologique qui cherche à intégrer les dimensions écologiques, économiques et sociales dans les réflexions sur les problèmes d'environnement. Du fait de ces influences multiples les auteurs, comme Clive Spash (2000) ou Martin O'Connor (2000), ayant adopté cette perspective d'analyse, sont ceux développant la critique la plus complète sur la MEC. O'Connor (2000: 177) insiste par exemple sur les incertitudes systémiques, les conflits distributifs et la diversité des positions morales et politiques inhérents aux problèmes d'environnement pour mettre en évidence les faiblesses théoriques de la MEC et de l'analyse coût bénéfice.

\section{Conclusion}

Malgré l'existence de doutes importants sur la fiabilité des réponses recueillies en univers hypothétique, malgré le développement d'analyses empiriques montrant que les réponses des individus à des questions de CAP sont fortement dépendantes du contexte et ne correspondent pas forcément à l'information recherchée et en dépit des réflexions philosophiques et théoriques remettant en cause l'idée même qu'il est possible de mesurer le prix de biens non marchands, l'histoire de la MEC est une «success story». Elle s'est imposée, en cinquante ans, comme la méthode permettant de mesurer la valeur totale des bien environnementaux dans la plupart des cadres réglementaires. Les économistes ont ainsi répondu à l'objectif qui leur était donné: un prix existe aujourd'hui pour la plupart des biens environnementaux, aisément disponible dans des bases de données accessibles sur internet.

En proposant une histoire de cet outil nous avons identifié plusieurs éléments expliquant ce succès. Conçue comme une méthode proposant d'associer préservation des milieux et régulation marchande, la MEC s'inscrit tout d'abord parfaitement dans le paradigme économique néolibéral initié dés la fin des années 70 et porté par les administrations Reagan et Tatcher, ou par la Banque Mondiale post - «consensus de Washington », qui furent les premières institutions ayant reconnu et légitimé la méthode.

L'aller-retour des légitimations entre la sphère politico-administrative et les milieux académiques est également déterminant, ces derniers apportant la caution finale de validité de la méthode. Mais ce brevet de scientificité ne peut s'apprécier sans avoir porté un regard sur la configuration de la science économique depuis quelques décennies et sur le peu de place qu'elle fait aux analyses sortant de l'orthodoxie néoclassique et de sa confiance dans les mécanismes de marché (voir par exemple Postel (2011) pour une analyse sur la France). Cet absence de pluralisme se retrouve dans la composition des comités d'experts, dans l'absence fréquente de références aux critiques de fond sur la MEC dans les rapports rédigés pour les pouvoirs publics, voire dans la 
nature même des questions auxquelles doivent répondre ces comités. A titre d'exemple, le panel d'expert du NOAA, qui faisait office d'instance supérieure de légitimation scientifique et qui s'enorgueillissait pour cela de la présence de deux «prix Nobel », n'avait pas à se prononcer sur une question aussi centrale que la validité du concept de «valeur d'existence » (Portney 1994) qui est pourtant fermement contestée par les analyses classique, marxiste ou institutionnelle.

Associée au climat idéologique, cette configuration de la discipline économique créé une boucle auto-référentielle sur laquelle repose la légitimité de la MEC: l'apparente unanimité des économistes sur le bien fondé de la méthode donne une légitimité scientifique au projet politique visant à intégrer la gestion des biens naturels dans un cadre marchand, et ce projet politique légitime à son tour la MEC en faisant de la production d'évaluations monétaires des biens naturels une nécessité primordiale («il faut donner un prix à la nature pour la protéger»).

Ce succès doit-il pour autant signifier la fin de la réflexion sur la MEC? Il nous semble que non, car il soulève au moins deux questions importantes. La première concerne la légitimité démocratique des décisions prises à l'aide de cette méthode. Si les analyses critiques à son sujet sont pertinentes et qu'elle ne mesure donc pas ce pour quoi elle a été imaginée, si, autrement dit, les chiffres utilisés par les décideurs n'ont pas le sens qui leur est prêté, la légitimité des décisions qu'elle supporte peut en effet être fortement remise en question. Pour lever ce doute, la recherche sur la méthode doit être poursuivie, mais hors du carcan théorique dans laquelle elle est enfermée depuis son invention, en laissant ouverte la possibilité que les questions de CAP ou CAR ne mesurent pas des variations de bien-être des individus. L'objectif de ces travaux devra être de comprendre la façon dont les individus construisent leur réponse et le sens qu'ils leur donnent. Ces recherches devront pour cela s'articuler autour du support de paiement (taxe, contribution à un programme de préservation, droit d'entrée dans un parc, cotisation à une association environnementale, etc.) qui est l'objet central de la MEC et qui est pourtant le grand absent des débats scientifiques la concernant (Milanesi 2009).

La deuxième question ne concerne plus l'outil et sa validité, elle prend acte du fait que des chiffres sont produits puis interprétés et utilisés comme la valeur monétaire de biens d'environnement, elle consiste plutôt à s'interroger sur le sens à donner à cette nouveauté. Nous observons ici un processus performatif (Muniesa et Callon 2009) où les économistes, par l'usage des outils tels que la MEC, se font les architectes d'une réalité sociale jusque là inconnue: ce qui n'avait pas de prix commence à en avoir un. Faut-il y voir, en suivant Polanyi, un processus violent et corrupteur, issu de la séparation radicale entre l'objet et le sujet qui selon Michel Aglietta et André Orléan (2002 : 43) fonde le modèle standard de l'échange marchand, ou assiste-t-on plutôt, comme a pu le décrire Viviana Zelizer (2011) sur l'assurance vie aux États-Unis, a une expression monétaire 
réappropriée par les acteurs et marquée par le social ?

\section{Références}

Ackerman, Frank et Lisa Heinzerling. 2004. Priceless. On knowing the price of everything and the value of nothing. New York, The New Press.

Aglietta, Michel et André Orléan. 2002. La monnaie entre violence et confiance. Paris, Odile Jacob.

Arrow, Kenneth et al.. 1993. «Report of the NOAA Panel on Contingent Valuation», Federal Register, vol. 58, n¹0: 4601-4614.

Amigues, Jean-Pierre, Fabienne Arnaux et François Bonnieux. 2003. Évaluation des dommages dans le domaine de l'eau: contribution à la constitution d'une base de donnée française. Paris, INRA.

Baumstark, Luc. 2004. La construction de valeurs socio-économiques environnementales : l'économiste dans la posture du passeur. Communication aux Journées AFSE Rennes, 18-19 mai 2004.

Bonnieux, François et Pierre Rainelli. 1999. «Contingent valuation methodology and the EU institutional framework», in Ian Bateman et Kenneth Willis (éd.), Valuing environmental preferences : theorie and practice of the contingent valuation method in the US, EC, and developing countries. Oxford, University press : 585-612.

Briscoe, John et al. 1990. «Toward equitable and sustainable rural Water supplies: a contingent valuation study in Brazil », World Bank Economic Review, vol. 4, n²: 115-134.

Carson, Richard T. et al. 1992. A Contingent Valuation Study of Lost Passive Use Values Resulting from the Exxon Valdez Oil Spill. A Report to the Attorney General of the State of Alaska.

Chevassus-au-Louis, Bernard et al. 2009. Approche économique de la biodiversité et des services liés aux écosystèmes Contribution à la décision publique. Paris, La documentation française (Centre d'analyse stratégique, 18).

Cummings, Ronald G., David S. Brookshire et William D. Schulze (éd.). 1986. Valuing Environmental Goods: A State of the Arts Assessment of the Contingent Method. Totowa, Rowman and Allanhed.

Diamond, Peter A. et Jerry A. Hausman. 1993. «On Contingent Valuation measurement of nonuse values », in Jerry A. Hausman (éd.), Contingent Valuation - A critical Assesment. Amsterdam, North Holland: 3-38.

Diamond, Peter A. et Jerry A. Hausman. 1994. «Contingent Valuation : Is some member 
better than no number? », Journal of economic perspectives, vol. 8, n4: 45-64.

Grandstaff, Somluckrat et John Dixon. 1986. «Evaluation of Lumpinee Park in Bangkok, Thailand», in John Dixon et M. Hufschmidt (éd.), Economic valuation techniques for the Environment: a case study workbook. Baltimore, The John Hopkins University Press : 121-140.

Grasso, Marco et Stefano Pareglio. 2002. Environmental valuation in European Union policy-making. Working paper. http://ideas.repec.org/p/wpa/wuwpot/0211001.html.

Grove-White, Robin. 1997. «The Environmental Valuation Controversy, Observations on its recent history and significance », in John Foster (éd.), Valuing Nature? Economics, Ethics and Environment. London, Routledge: 21-31.

Hanemann, Michael W. 1994. «Valuing the environment through contingent valuation », Journal of economic perspectives, vol. 8, $\mathrm{n}^{\circ}$ 4: 19-43.

Hausman, Jerry A. 1993. Contingent Valuation - A critical Assesment. Amsterdam, North Holland.

Hausman, Jerry A., Gregory K. Leonard et Daniel McFadden. 1995. «A Utility-consistent, Combined Discrete Choice and Count Data Model Assessing Recreational Use Losses Due to Natural Resource Damage », Journal of Public Economics, vol. 56: 1-30.

Hodgson, Geoffrey. 1997. «Economics, environmental policy and the transcedence of utilitarism », in John Foster (éd.), Valuing Nature? Economics, Ethics and Environment. London, Routledge: 48-63.

Kahneman, Daniel et Ilana Ritov. 1994. «Determinants of stated willingness to pay for public goods: a study in the headline method », Journal of Risk and Uncertainty, vol. 9: 5-38.

Kahneman, Daniel et Jack Knetsch. 1992. «Valuing public goods: The purchase of moral satisfaction », Journal of Environmental Economics and Management, vol. 22: 57-70.

Latour, Bruno. 1997 [1991]. Nous n'avons jamais été modernes: essai d'anthropologie symétrique. Paris, La Découverte.

Loomis, John B. 1999. «Contingent Valuation Methodology and the US Institutional Framework », in Ian Bateman et Kenneth Willis (éd.), Valuing environmental preferences : theorie and practice of the contingent valuation method in the US, EC, and developing countries. Oxford, University press: 613-627.

Milanesi, Julien. 2007. « La méthode d'évaluation contingente en question. Critique, requalification et illustration par la mesure de la demande en assainissement à Moshi (Tanzanie) », Thèse de doctorat, Université de Pau et des Pays de l'Adour.

Milanesi, Julien. 2009. (ré)Interpréter les réponses aux enquêtes d'évaluation contingente : du malentendu à l'analyse du compromis. Communication aux Doctoriales GDR CNRS «Économie 
\& sociologie », Université de Lille I, Clersé, 20 novembre 2009.

Milanesi, Julien. 2010. «Ethique et évaluation monétaire de l'environnement. La nature est elle soluble dans l'utilité ?», VertigO, vol. 10, n², en ligne.

Ministère de l'Ecologie et du Développement Durable. 2003. L'évaluation économique des biens et dommages environnementaux. Actes du colloque du 22 mai 2003, non publié.

Ministère de l'équipement, des transports, du logement, du tourisme et de la mer. 2005. Instruction cadre relative aux méthodes d'évaluation économique des grands projets d'infrastructures de transport du 25 mars 2004, actualisée le 27 mai 2005.

Mitchell, Robert C. et Richard. T. Carson. 1989. Using surveys to value public goods: the contingent valuation method. Washington DC, Resources for the future.

Muniesa, Fabian et Michel Callon. 2009. «La performativité des sciences économiques», in Philippe Steiner et François Vatin (éd.), Traité de sociologie économique. Paris, PUF: 289-324.

Navrud, Stale et Mette Vagnes. 2000. Assessment of Environmental Valuation Reference Inventory (EVRI) and the Expansion of Its Coverage to the EU. Report to the European Commission, DG XI.

Navrud, Stale et Gerald J. Pruckner. 1997. «Environmental valuation - To use or not to use? A comparative study of the United States and Europe», Environmental and resource economics, vol. $10, \mathrm{n}^{\circ} 1: 1-26$.

O'Connor, Martin. 2000. «Pathways for environmental evaluation: a walk in the (hanging) garden of Babylon », Ecological Economics, vol. 34: 175-193.

O'Neill, John. 1997. «Value, pluralism, incommensurability and institutions », in John Foster (éd.), Valuing Nature? Economics, Ethics and Environment. London, Routledge:75-88.

Portney, Paul R. 1994. «The Contingent Valuation Debate: Why Economist Should care?», Journal of economic perspectives, vol. 8, n4: 3-17.

Postel, Nicolas. 2011. «Le pluralisme est mort, vive le pluralisme », L'Economie politique, $n^{\circ} 50: 6-31$.

Randall, Alan. 1998. «Beyond the crucial experiment: mapping the performance characteristics of contingent valuation », Resources and energy Economics, vol. 20, n²:197-206.

Sagoff, Mark. 2004. Price, Principle, and the Environment. Cambridge, University Press.

Schkade, David A. et John W. Payne. 1993. «Where do the number come from? How people respond to contingent valuation questions », in Jerry A. Hausman (éd.), Contingent Valuation - A critical Assesment. Amsterdam, North Holland: 271-303.

Spash, Clive. 2000. «Ecosystems, contingent valuation and ethics: the case of wetland recreation », Ecological Economics, vol. 34: 195-215. 
Stern, Nicolas. 2007. The economics of climate change. Cambridge, University Press.

Stevens, Thomas et al. 1991. «Measuring the existence value of wildlife: what do CVM estimates really show », Land Economics, vol.67, n4: 390-400.

Sukhdev, Pavan (éd.). 2008. L'économie des écosystèmes et de la biodiversité. Rapport d'étape, Communauté Européenne.

Terra, Sebastien. 2005. «Guide de bonnes pratiques pour la mise en œuvre de la méthode d'évaluation contingente », Série Méthode, Document de travail, MEDD, D4E, n $05-\mathrm{M} 04$.

Thompson, Dale B. 2002. «Valuing the environment, courts struggles with natural ressource damages », Environmental Law, vol.32: 57-89.

Touaty, Myriam et Gérard Gié. 2004. Synthèse des travaux français relatifs à l'évaluation des dommages. Contribution au 10ème colloque de comptabilité nationale organisé par l'ACN à Paris les 21, 22 et 23 janvier 2004.

Tversky, Amos et Richard Thaler. 1990. «Preference Reversals », The Journal of Economic Perspectives, vol.4, $\mathrm{n}^{\circ}$ 2: 201-211.

Venkatachalam, L. 2004. «The contingent valuation method: a review », Environmental impact assesment review, vol. 24: 89-224.

WASH. 1988. «Guidelines for Conducting Willingness-to-Pay Studies for Improved Water Services in Developing Countries », Field Report, $\mathrm{n}^{\circ} 306$.

Whittington, Dale et al. 1990. «Estimating the willingness to pay for water services in developing countries: a case study of contingent valuation in southern Haïti », Economic development and cultural change, vol. $38, \mathrm{n}^{\circ} 2:$ 293-311.

World Bank Water Demand Research Team. 1993. «The demand for water in rural areas: determinants and policy implications », World Bank Research Observer, vol. 8, n 1: 47-70.

Zelizer, Viviana. 2011. Economic Lives. How culture dhape the economy? Princeton \& Oxford, Princeton University Press.

i Le propos est de Pavan Sukhdev, économiste, banquier et responsable de l'initiative internationale sur «L'économie des écosystèmes et de la biodiversité» (http://www.3dterritoires.org/environnement/biodiversite/pavan-sukhdev—ilfaut-donner-un-prix-a-la-biodiversite.html).

ii En opposition à la notion de «valeur d'usage » (ou « use value »), qui serait propre à un usage direct ou indirect du bien, ce concept de «valeur de non-usage » repose sur les travaux de Krutilla et Weisbrod qui proposaient dans les années 60 de considérer la valeur que les individus attribuent à l'existence même des biens d'environnement ou à la possibilité de pouvoir en avoir l'usage dans le futur (Stevens et alii 1991: 390). Dans ce cadre théorique, la notion de «valeur d'usage » prend un sens différent de celui définit dans la tradition de l'économie politique classique ou marxiste. 
iii Les externalités négatives, ou « coûts externes », sont dans leur acception la plus courante les coûts générés par un producteur sur un tiers qui ne sont pas pris en compte par le marché.

iv Le projet WASH ("Water and Sanitation for Health", 1981-1994) fut un élément de la participation du gouvernement étasunien à la décennie internationale sur l'eau des Nations Unies. (voir http://www.usaid.gov/our_work/global_health/eh/ehhistory.html)

v Richard Carson et Robert Mitchell, qui avaient publié l'ouvrage de référence sur la MEC en 1989, sont deux des auteurs de cette étude.

vi La méthode d'analyse conjointe (ou des choix expérimentaux) est, comme la MEC, fondée sur les préférences déclarées d'individus interrogés lors d'une enquête, ses fondements théoriques sont également les mêmes. Elle consiste à faire choisir les enquêtés entre plusieurs alternatives aux attributs différents.

vii Ces différentes études sont consultables sur le site du Ministère de l'écologie : http://www.developpementdurable.gouv.fr/ 